\title{
Il Miele Medicale nella pratica clinica: la nostra esperienza in 75 casi trattati
}

\author{
Marina Pierangeli, Giovanni Ruocco, Vanya Recchi, Valentina Cecconato, Costanza Binci, Yasmine Zavalloni, \\ Davide Talevi, Matteo Torresetti, Giovanni Di Benedetto, Alessandro Scalise \\ Struttura Organizzativa Dipartimentale S.O.D., Clinica di Chirurgia Plastica e Ricostruttiva, Azienda Ospedaliero-Universitaria \\ Ospedali Riuniti di Ancona, Torrette (AN), Italia
}

\begin{abstract}
RIASSUNTO
Presso la Struttura Organizzativa Dipartimentale (S.O.D.) Clinica di Chirurgia Plastica e Ricostruttiva di Ancona sono stati trattati con miele medicale consecutivamente, nell'arco di 24 mesi, 75 pazienti affetti da lesioni cutanee croniche. Nell'articolo vengono descritti gli ottimi risultati ottenuti in termini di riduzione dei tempi di guarigione delle lesioni trattate, e vengono descritte le caratteristiche chimiche e fisiche del miele medicale che ne spiegano in forma scientifica l'azione.
\end{abstract}

\section{INTRODUZIONE}

Il miele è conosciuto come potente antibatterico ed antiinfiammatorio fin dall'antichità. Ad esempio nella tomba di Pabusa a Luxor, risalente al 600 a.C., è raffigurato un apicoltore egizio che preleva il miele. Nel Papiro di Ebers, risalente al 1550 a.C., sono elencate molte sue possibili utilizzazioni (fino a 147), ed in particolare il miele viene descritto per il trattamento di ustioni, piaghe e per velocizzare il processo di guarigione dopo interventi chirurgici, soprattutto in caso di circoncisione. ${ }^{1,2}$

Con l'avvento degli antibiotici il miele è stato progressivamente abbandonato, fino a ritrovare nuova vita negli ultimi anni come prodotto naturale utilizzabile per prevenire e per trattare i casi con sviluppo di infezioni da germi multiresistenti. ${ }^{3,4}$

Corrispondenza: Marina Pierangeli, Specialista Chirurgia Plastica e Ricostruttiva, Dirigente Medico a tempo indeterminato presso S.O.D. Clinica di Chirurgia Plastica e Ricostruttiva, Azienda Ospedali Riuniti di Ancona, Via Conca 71, 60100 Ancona, Italia Tel.: 071-5963500. Fax: 071-5963486.

E-mail: marina.pierangeli@ospedaliriuniti.marche.it

Conflitti di interesse: Gli autori dichiarano l'assenza di conflitti di interesse.

Parole chiave: Medical grade honey; wound hard to heal; wound dressing.

Ricevuto per la pubblicazione: 18 febbraio 2020.

Accettato per la pubblicazione: 9 aprile 2020 .

This work is licensed under a Creative Commons Attribution NonCommercial 4.0 License (CC BY-NC 4.0).

${ }^{\circ}$ Copyright: the Author(s), 2020

Licensee PAGEPress, Italy

Italian Journal of Wound Care 2020; 4(1):23-28

doi:10.4081/ijwc.2020.58
In commercio esistono diversi tipi di miele, ma nella nostra clinica abbiamo avuto la possibilità di utilizzare il miele medicale Medical Grade Honey.

Nella nostra Clinica abbiamo trattato in 24 mesi 75 pazienti affetti da lesioni cutanee croniche contaminate $\mathrm{e}$ infette, di diversa eziopatogenesi: lesioni di origine vascolare venosa, arteriosa, mista, lesioni post traumatiche, lesioni diabetiche, lesioni post chirurgiche, lesioni da pressione ed ustioni.

In alcuni casi la terapia con miele medicale è stata affiancata da altri trattamenti (es. trattamento biofotonico o Negative Pressure Wound Therapy, NPWT), ottenendo in ogni caso un buon effetto sulla velocizzazione dei processi di guarigione.

\section{MATERIALI E METODI}

Nell'arco di 24 mesi sono stati trattati presso la Struttura Organizzativa Dipartimentale (S.O.D.) Clinica di Chirurgia Plastica e Ricostruttiva di Ancona 75 pazienti. L'età media dei pazienti era 66,64 anni; le lesioni avevano un'età di insorgenza di 5,7 mesi.

La tipologia delle lesioni comprendeva: 3 Lesioni Da Pressione (LDP), 15 lesioni post chirurgiche (diastasi, skin tears, mancato attecchimento di innesti cutanei, medicazione di aree prelievo di innesti cutanei), 24 lesioni post traumatiche (abrasioni, ferite a lembo, ferite lacero contuse, necrosi cutanee), 9 ustioni di I-II grado, 23 lesioni vascolari di cui 15 ulcere venose, 4 ulcere arteriose e 5 ulcere miste (Figura 1).

Di queste lesioni 42 erano hard to heal, soprattutto tutte le lesioni da pressione e le lesioni vascolari arteriose e miste. Il 53\% delle lesioni presentavano segni clinici di colonizzazione batterica ed i risultati dei prelievi colturali mostravano nel $32 \%$ di questi casi la crescita di batteri multiresistenti.

Le lesioni sono state misurate utilizzando un device 
non a contatto (Moleculight) al tempo T0, ed ad intervalli di 7 giorni; l'area media misurata al tempo T0 è stata di $76,8 \mathrm{~cm}^{2}$.

Il miele medicale che abbiamo utilizzato era in tre formati: gel, alginato + miele e pad contenente miele + collagene.

Il protocollo di trattamento ha previsto l'utilizzazione del formato in gel nei casi che presentavano un fondo di lesione ancora maldeterso, con segni di contaminazione/colonizzazione, con margini non in riepitelizzazione: il gel, previa disinfezione e detersione delle lesioni trattate, veniva applicato sul fondo di lesione $1 \mathrm{v} / 2 \mathrm{gg}$, generalmente in piccole quantità poiché la sua consistenza rendeva possibile distribuirlo molto agevolmente su tutta le lesione, ed associandolo ad altre medicazioni secondarie (es. garza non ad e garze sterili e bendaggio elastocompressivo).

Il miele medicale in formato alginato + miele è stato utilizzato prettamente in lesioni essudanti, cavitarie e sottominate, con segni di contaminazione/colonizzazione batterica, e prevedeva un cambio di medicazione ogni 2 giorni previa disinfezione e detersione della lesione; questo formato ha previsto anche l'utilizzazione di medicazioni secondarie, tipo garze non $a d$, schiume di poliuretano e garze sterili.

Il miele medicale in formato pad con collagene prevedeva, invece, il suo utilizzo nei casi in cui il processo di guarigione era stato riavviato, pertanto in lesioni che, seppur con segni di colonizzazione/contaminazione batterica, mostravano un fondo di lesione tendenzialmente granuleggiante e con margini in riepitelizzazione, ma che necessitavano di un'ulteriore spinta del processo rigenerativo, ottenendo anche un sollevamento/riempimento del fondo di lesioni particolarmente profonde. La medicazione, previa disinfezione e detersione della lesione, veniva eseguita ogni 3-4 giorni, associando il pad a medicazioni secondarie, tipo garze non ad e garze sterili.

I tempi medi di utilizzazione del miele medicale in tutte le lesioni trattate sono stati di 28 giorni, con un minimo di 7 giorni ed un massimo di 90 giorni.

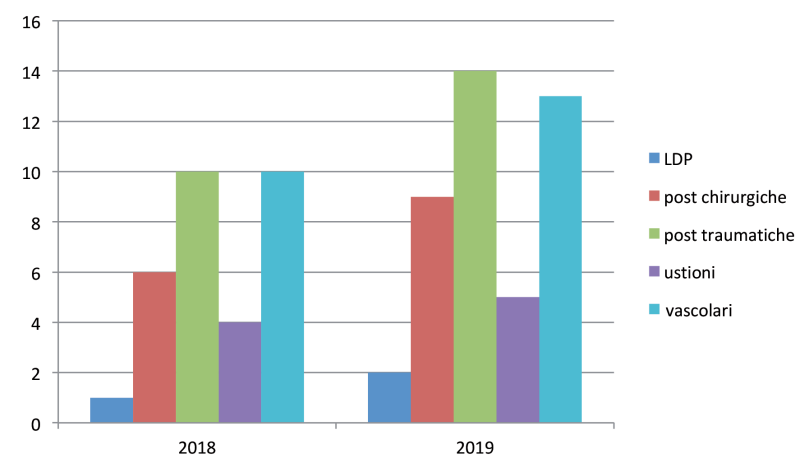

Figura 1. 75 casi di Lesioni Cutanee Croniche (LCC) trattati in 24 mesi suddivisi per classi.
Tutte le lesioni trattate si sono dimostrate responsive al miele medicale, con riduzione degli essudati, del dolore, dell'iperemia e dell'edema dei tessuti perilesionali.

Il $72 \%$ delle lesioni è andato incontro a completa guarigione utilizzando diverse tipologie di miele medicale (in forma di gel, di alginato + miele, di pad contenente miele + collagene), il 15\% delle lesioni è guarito associando il miele medicale ad altri trattamenti (es. biofotonica o NPWT), il 10\% delle lesioni è migliorato a livello di qualità del fondo delle ferite, rendendo possibile utilizzare tecniche chirurgiche di chiusura (es. innesti cutanei liberi), il restante $3 \%$ delle lesioni non ha tratto vantaggio dall'utilizzazione di tale prodotto (Figure 2-6).

Il miele medicale non ha provocato in nessuno dei casi trattati effetti collaterali, e tutti i pazienti hanno tollerato la medicazione.

Va anche considerata l'estrema facilità di utilizzo del miele medicale nei tre formati che abbiamo avuto a disposizione, pertanto i pazienti sono stati trattati in maniera molto semplice anche a domicilio da parte del care giver.

\section{DISCUSSIONE}

Nel trattamento delle Lesioni Cutanee Croniche (LCC) e delle lesioni post traumatiche che sono giunte alla nostra osservazione e che abbiamo sottoposto a terapia con miele medicale, abbiamo notato un notevole miglioramento in termini di riduzione dei tempi necessari alla guarigione delle lesioni e in termini di riduzione delle complicanze legate alla contaminazione/colonizzazione batterica.

Abbiamo quindi effettuato una revisione della letteratura riguardante il miele medicale per capire i motivi che hanno determinato una così rapida riduzione delle infezioni ed una guarigione così repentina delle lesioni da noi trattate.

Il miele naturale è composto per il $38,5 \%$ da fruttosio, $31 \%$ da glucosio, $12,9 \%$ da altri zuccheri; contiene inoltre il $17,1 \%$ di acqua, e lo $0,5 \%$ di proteine, acidi organici, multiminerali, amminoacidi, vitamine, fenoli e altri composti. ${ }^{5}$ Fin dall'antichità ne sono riconosciute le proprietà biologiche, fisiologiche e farmacologiche ${ }^{1}$ oltre che energetiche e funzionali.

$\mathrm{Ci}$ sono evidenze in letteratura riguardo la potente attività in vitro ${ }^{6}$ del miele contro batteri resistenti agli antibiotici, soprattutto E. Coli ML-35, Ps. Aeruginosa PAO1, Enterobacter Cloacae, Klebsiella Oxytoca, St. Epidermidis, St. Aureus ed Enterococcus Faecium. ${ }^{7,8}$

La spiegazione dell'attività antibatterica del miele si basa su diversi fattori.

Intanto il miele ha una importante attività osmotica legata al suo alto contenuto in zuccheri, che comporta il blocco della crescita dei batteri favorendo la guarigione: il miele infatti, essendo una sostanza igroscopica, tende 


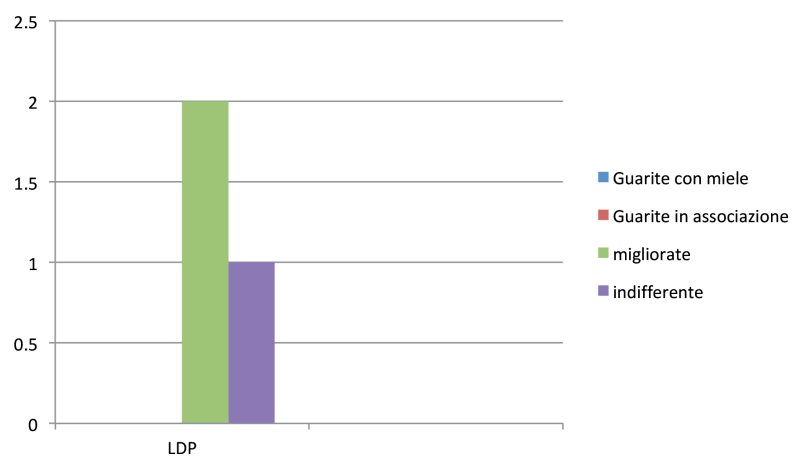

Figura 2. 3 casi di Lesioni Da Pressione (LDP). Numero di casi guariti utilizzando solo il miele medicale, o in associazione con alter medicazioni; numero di casi migliorati; numero di casi che non hanno trovato giovamento dal trattamento.

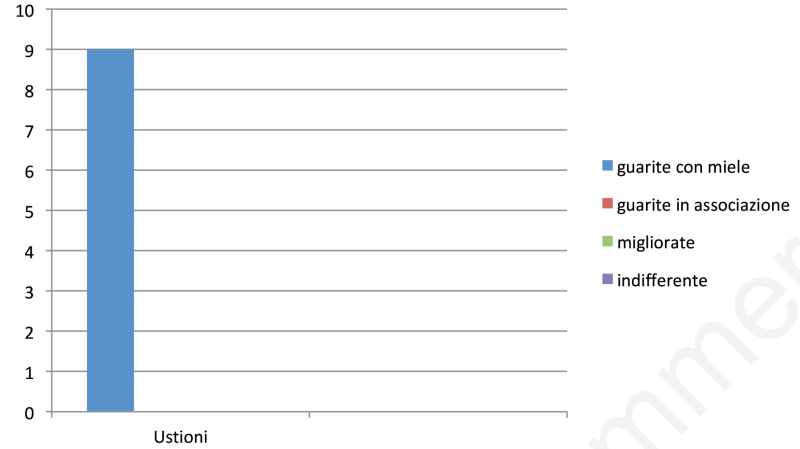

Figura 3. 9 casi di ustione. Numero di casi guariti utilizzando solo il miele medicale, o in associazione con alter medicazioni; numero di casi migliorati; numero di casi che non hanno trovato giovamento dal trattamento.

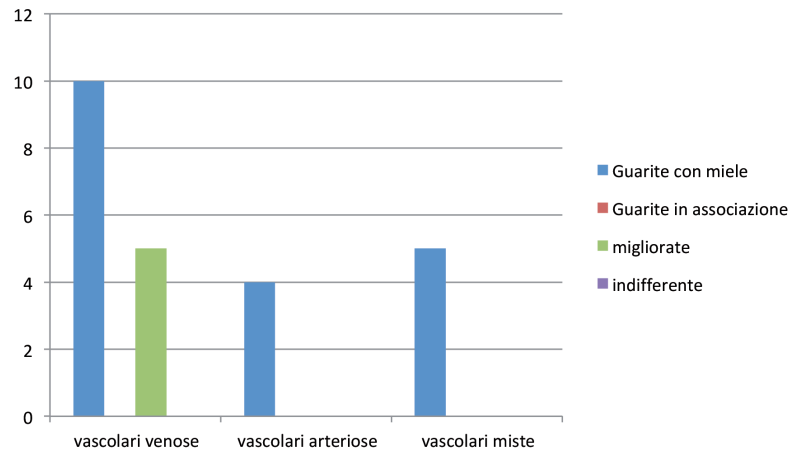

Figura 4. 23 casi di lesioni vascolari. Numero di casi guariti utilizzando solo il miele medicale, o in associazione con alter medicazioni; numero di casi migliorati; numero di casi che non hanno trovato giovamento dal trattamento. ad estrarre l'umidità dall'ambiente batterico, portando i batteri stessi a disidratazione. ${ }^{9}$ Tuttavia, anche se il miele viene molto diluito in acqua, riducendo l'effetto osmotico, presenta ancora attività antibatterica. ${ }^{9-11}$ Sembra, quindi, che l'attività antibatterica del miele sia legata ad altri fattori, ovvero alla presenza di perossido di idrogeno e di acido gluconico che vengono continuamente prodotti dall'enzima Glucosio ossidasi (GOX) presente, in particolare, nel miele medicale Medical Grade Honey. La concentrazione del perossido di idrogeno resta costante allo $3 \%$ per 48 ore dopo la sua applicazione sulle ferite, e contribuisce a contrastare $\mathrm{i}$ batteri patogeni, mentre l'acido gluconico mantiene un ambiente acido sulla ferita, aiutando ad inibire la proliferazione batterica. ${ }^{6} \dot{\mathrm{E}}$ infatti noto che il miele ha un $\mathrm{pH}$ medio di $4,4,{ }^{12,13}$ pertanto se applicato sul fondo di una ferita, riesce a mantenere acido il

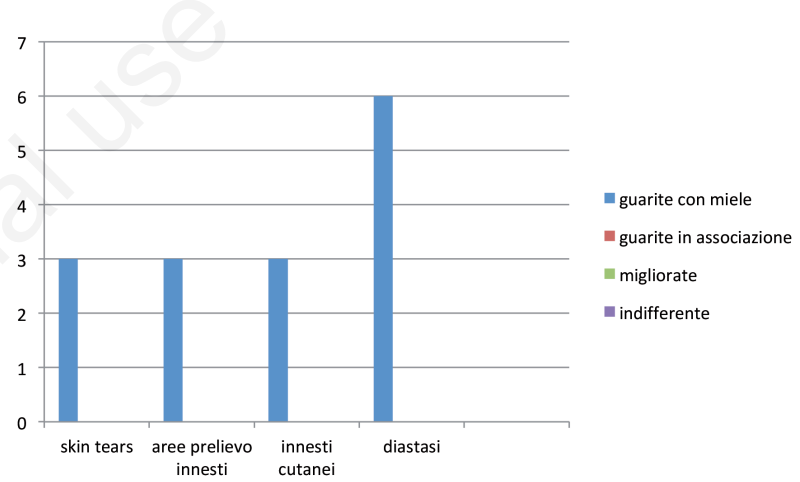

Figura 5. 15 casi di lesioni post chirurgiche. Numero di casi guariti utilizzando solo il miele medicale, o in associazione con alter medicazioni; numero di casi migliorati; numero di casi che non hanno trovato giovamento dal trattamento.

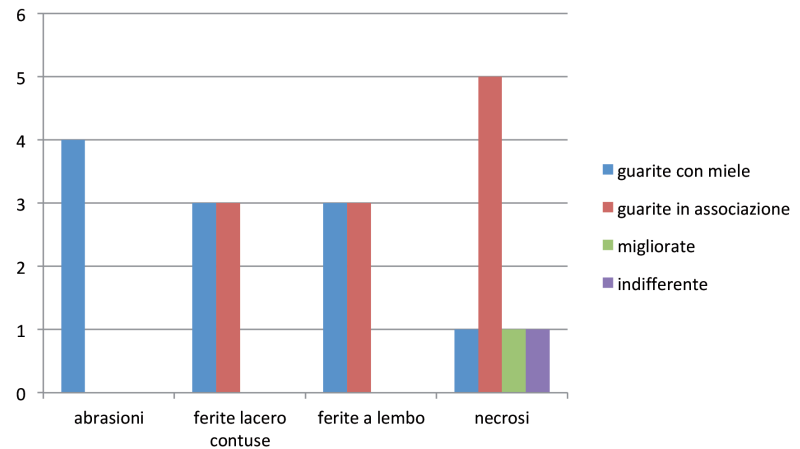

Figura 6. 24 casi di lesioni post traumatiche. Numero di casi guariti utilizzando solo il miele medicale, o in associazione con alter medicazioni; numero di casi migliorati; numero di casi che non hanno trovato giovamento dal trattamento. 
microambiente, accelerandone la guarigione e riducendo il rischio di colonizzazione o infezione batterica, che sono situazioni cliniche che si verificano quando il $\mathrm{pH}$ medio è maggiore di 7,3, soprattutto in presenza di essudato. ${ }^{12-14}$

Altre tipologie di mele medicale (es. il miele di Manuka) contengono un altro composto antibatterico metilgliossale (MGO), che è un metabolita reattivo che deriva dalla conversione di diidrossiacetone (DHA), e che è in grado di impedire la sintesi delle proteine, in particolare dell'RNA e del DNA anche batterico. ${ }^{15,16}$

Un altro importante composto antibatterico presente nel miele medicale è la defensina 1 , che è stata isolata per la prima volta nella pappa reale, ${ }^{17}$ prodotta dalle secrezioni delle ghiandole ipofaringee e mandibolari delle giovani api operaie. ${ }^{18,19}$ Quando poi le api invecchiano, non producono più la pappa reale, ma diventano produttrici di miele: tuttavia le loro ghiandole continuano a secernere la defensina 1, la quale, con il perossido di idrogeno e l'acido gluconico, sembra avere un importante ruolo nel contribuire a mantenere il miele acerbo contro il deterioramento microbico. ${ }^{8,12}$

Il miele medicale possiede anche proprietà antiinfiammatorie, soprattutto legate alle caratteristiche igroscopiche del miele, che tende quindi a diminuire la produzione di edemi e di essudati: questa attività tende a ridurre anche il dolore causato dalla pressione sulle terminazioni nervose e riduce la produzione di prostaglandine, che iniziano e mantengono il processo flogistico. ${ }^{10} \mathrm{Il}$ dolore e l'edema comunque sono attenuati anche grazie all'azione stimolante del miele sulla produzione di tessuto di granulazione, sull'angiogenesi e sulla epitelizzazione: ${ }^{20}$ tale attività potrebbe essere legata al mantenimento di un $\mathrm{pH}$ acido all'interno della ferita, che è la principale causa di un aumentato rilascio di ossigeno dall'emoglobina all'interno dei capillari.

Abbiamo quindi rilevato delle fondamentali differenze tra il miele naturale, quello da noi utilizzato (Medical Grade Honey), e quello prodotto dal polline del Leptospermum Scoparium (miele di Manuka) (Tabella 1).

In particolare, il miele di Manuka è l'unico a possedere il MGO: chiamato anche piruvaldeide, è un prodotto organico derivato dall'acido piruvico, che si forma nel metabolismo dello zucchero, e che si può trovare all'interno di cibi e bevande.
Tuttavia dalla letteratura risulta che tale prodotto è citotossico non solo nei riguardi delle cellule batteriche, ma anche nei riguardi dell'ospite. Pertanto questo miele, anche se presenta delle moderate caratteristiche antibatteriche, in casi di ulcere diabetiche rallenta addirittura il processo di guarigione. ${ }^{21}$

Il miele medicale Medical Grade Honey invece, possiede la GOX e la defensin-1 peptide.

La prima sostanza determina una produzione di perossido di idrogeno al 3\%, ovvero in concentrazioni non citotossiche, bensì battericide per un tempo fino a 48 ore dopo l'applicazione del miele sulle lesioni, e di Acido Gluconico, che possiede un importante potere battericida in quanto determina il mantenimento di un $\mathrm{pH}$ acido del miele.

La defensina-1, prodotta dalle ghiandole salivari delle api produttrici di pappa reale e di miele, ha un forte effetto battericida, rapido ed ad ampio spettro. ${ }^{6} \mathrm{Il}$ supposto meccanismo di azione è l'attacco diretto alle proteine transmembrana caricate negativamente presenti sulla membrana microbica, ${ }^{22}$ che provoca o la distruzione della membrana, o la migrazione di dette proteine all'interno della cellula batterica. ${ }^{23,24}$ Alcuni batteri sembrano aver sviluppato un meccanismo per ridurre la loro suscettibilità alla defensina-1, riducendo il numero delle proteine transmembrana. Tuttavia non si prevede una rapida diffusione di tale resistenza (che porterebbe ad un ridotto effetto battericida del miele) perché sono coinvolti meccanismi genetici molto complessi. ${ }^{25}$

Il miele possiede anche un'azione antiossidante legata alla presenza nella sua composizione di flavonoidi, monofenoli, polifenoli e Vitamina $C,{ }^{26,27}$ che vanno a ridurre gli effetti lesivi dei Reactive Oxygel Species (ROS).$^{28}$ Questi antiossidanti presenti nel miele hanno qualità sia idrofile che lipofile, andando ad agire a diversi livelli cellulari. ${ }^{29}$

Il miele medicale entra in gioco anche come immunostimolante: esso infatti stimola sia la produzione della $\mathrm{TNF} \alpha$, attivando i macrofagi, stimolando l'angiogenesi e la collagenasi, sia la produzione della IL-6, che inibisce la generazione di TNF $\alpha$ e che stimola la riepitelizzazione. ${ }^{30}$

Altra attività del miele medicale ormai documentata scientificamente, è quella di promuovere il debridement del fondo delle ferite: in particolare, ci sono evidenze che il miele applicato per via topica sulle ustioni, impedendo

Tabella 1. Principali caratteristiche delle differenti tipologie di miele medicale in commercio.

\begin{tabular}{|c|c|c|c|c|c|}
\hline \multirow[t]{2}{*}{ Tipologia di miele } & \multicolumn{5}{|c|}{ Componenti antibatteriche e antiossidanti } \\
\hline & $\begin{array}{l}\text { Basso pH } \\
\text { alti valori di zucchero }\end{array}$ & $\begin{array}{c}\text { Alti valori } \\
\text { di antiossidanti }\end{array}$ & $\begin{array}{l}\text { Alto valore } \\
\text { di GOX }\end{array}$ & $\begin{array}{c}\text { Alto valore } \\
\text { del peptide } \\
\text { Bee-Defensin-1 }\end{array}$ & $\begin{array}{c}\text { Alto valore } \\
\text { di Methyl glyoxal }\end{array}$ \\
\hline Certified Organic & + & $?$ & $?$ & - & - \\
\hline Medical Grade & + & - & + & + & - \\
\hline Leptospermum & + & + & - & - & + \\
\hline
\end{tabular}


la formazione di un'escara, risultava superiore all'utilizzo di altri presidi, come ad esempio la sulfadiazina di argento o l'idrogel. ${ }^{31-33}$ La spiegazione di questa azione di debrider del miele medicale sta nello stimolare la produzione di plasmina (contrastando l'azione dell'Inibitore dell'Attivatore del plasminogeno, ovvero il PAI), che è un enzima che digerisce la fibrina ma non la matrice di collagene, pertanto elimina un prodotto dannoso che impedisce i normali processi di guarigione delle ferite senza interferire sulla componente rigenerativa. ${ }^{34}$

Il miele medicale Medical Grade Honey può essere utilizzato in sicurezza anche in ambiente pediatrico, grazie alla assenza di composti dannosi nella sua composizione. Pertanto è utilizzabile sia per il trattamento dell'eritema da pannolino, che in presenza di problemi di riepitelizzazione in sede di stomia.,35 Poiché il miele medicale è sterile e non contiene sostanze tossiche come il cortisone, può essere utilizzato in sicurezza per il trattamento delle ragadi al seno durante l'allattamento, in quanto, anche se ingerito, non reca danni al neonato durante la suzione.

Tutti i tipi di miele medicale sono sterilizzati ai raggi gamma, ma mantengono la loro attività antibatterica. ${ }^{36}$

\section{CONCLUSIONI}

In un'epoca in cui l'utilizzazione massiva ed incongrua degli antibiotici ha prodotto la selezione di ceppi batterici multiresistenti, si rende necessario ricorrere a soluzioni terapeutiche più naturali, con un'ottica però più moderna.

L'azione antibatterica, antifungina ed antiinfiammatoria, antiossidante, immunostimolante e di debridement del miele è stata ampiamente dimostrata da evidenze scientifiche con forza di raccomandazione elevata.

Non tutti i tipi di miele medicale hanno gli stessi effetti sulla guarigione delle lesioni cutanee croniche.

Questo diverso comportamento è spiegabile scientificamente.

In particolare è stato dimostrato che tra tutti i mieli medicali, il Medical Grade Honey possiede il più alto potere antiinfiammatorio ed antibatterico in quanto è l'unico a contenere il GOX e la defensina-1, e con livelli di sicurezza produttiva elevati che ne consentono l'utilizzo nell'adulto, nel neonato e nel mondo animale.

\section{BIBLIOGRAFIA}

1. Alvarez-Suarez JM, Tulipani S, Romandini S, et al. Contribution of honey in nutrition and human health: a review. Med J Nutrition Metab 2010;3:15-23.

2. Majino G. Man and wound in the ancient world. Cambridge: Harvard University Press; 1975.

3. Jones R. Honey and healing through the ages. In: Munn P,
Jones R. (eds.) Honey and Healing. Cardiff: International Bee Research Association;2001, pp.1-4.

4. Nathan C. Antibiotics at the crossroads. Nature 2004;431:899-902.

5. Pasupuleti VR, Sammugam R, Ramesh N, Gan SH. Honey, propolis and Royal Jelly: a comprehensive review of their biological actions and health benefits. Hindawi Oxidative Medicine and Cellular Longevity 2017;2017:1259510. doi: $10.1155 / 2017 / 1259510$.

6. Kwakman PH, de Boer L, Ruyter Spira CP, et al. Medicalgrade honey enriched with antimicrobial peptides has enhanced activity against antibiotic-resistant pathogenes. Eur J Clin Microbiol Infect Dis 2011;30:251-7.

7. Leher RI, Barton A, Daher KA, et al: Interaction of human defensins with Escherichia coli: mechanism of bacterial activity. J of Clin Investigation 1989;84:553-61.

8. Kwakmann PH, Van der Akker GP, Güçlü A, et al: Medicalgrade honey kills antibiotic-resistant bacteria in vitro and eradicates skin colonization. Cl Infect Dis 2008;46:1677-83.

9. Molan PC. The evidence supporting the use of honey as a wound dressing. Intern J Low Extrem Wounds 2006;5:40-54.

10. Simon A, Traynor K, Santos K, et al: Medical honey for wound care-still the "latest resort"? Evid Based Complement Alternat Med 2009;6:165-73.

11. Molan PC, Betts JA, Clinical usage of honey as a wound dressing. Intern J Low Extrem Wounds 2004;13:353-6.

12. Molan PC. The antibacterial activity of honey. Bee World 1992;73:5-28.

13. Aureli P, Franciosa G, Fenicia L. Infant botulism and honey in Europe: a commentary. Pediatric Infect Dis J 2002;21:866-8.

14. Schneider LS, Korber A, Grabbe S, Dissemond J. Influence of $\mathrm{pH}$ on wound healing: a new prospective for wound-therapy? Arch of Dermatol Res 2007;298:413-20.

15. Adams CJ, Boult CH, Deadman BJ, et al. Isolation by HPLC and characterisation of the bioactive fraction of New Zealand manuka (leptospermum scoparium) honey. Carbohydrate Res 2008;343:651-9.

16. Mavric E, Wittmann S, Barth G, Henle T. Identification and quantification of methylglyoxal as the dominant antibacterial constituent of Manuka (Leptosperum scoparium) honeys from New Zealand. Molecular Nutrition and Food Res 2008;52:483-9.

17. Fujivara S, Imai J, Fujivara M, et al. A potent antibacterial protein in royal jelly. Purification and determination of the primary structure of royalisin. J Biol Chem 1990;265:11333-7.

18. Lensky Y, Rakover Y. Separate protein body compartments of the worker honeybee (Apis mellifera L.). Comp Biochem Physiol 1983;75:607-15.

19. Knecht D, Kaatz HH. Patterns of larvel food-production by hypopharyngeal glands in adult worker honey-bees. Apidologie 1990;21:457-68.

20. Al-Waili NS, Saloom K, Butler G, Al Ghamdi AA. Honey and microbial infection: a review supporting the use of honey for microbial control. J Med Food 2011;14:1079-96.

21. Majtan J. Methylglyoxal-a potential risk factor of manuka honey in healing of diabetic ulcers. Evid Based Complement Alternat Med 2011;2011:295494.

22. Huang HV. Action of antimicrobial peptides: two-state model. Biochemistry 2000;39:8347-52. 
23. Epand RM, Vogel HJ. Diversity of antimicrobial peptides and their mechanisms of action. Biochim Biophys Acta 1999;1462:11-28.

24. Matzukaki K. Why and how are peptide-lipid interactions utilized for self-defense? Magainins and tachiplesins as archetypes. Biochim Biophys Acta 1999;1462:1-10.

25. Devine DA, Hancock HE. Cationic peptides: distribution and mechanisms of resistance. Curr Pharm Des 2002;8:703-14.

26. Bergman A, Yanai J, Weiss J, et al. Accerelation of wound healing by topical application of honey. An animal model. Am J Surg 1983;145:374-6.

27. van den Berg AJ, van der Worm E, et al. An in vitro examination of the anti-oxidant and anti-infiammatoy properties of buckwheat honey. J Wound Care 2008;17:172-4.

28. Bashkaran K, Zunaina E, Bakiah S, et al. Anti-inflammatory and antioxidant effect of tualang honey in alkali injury on the eyes of rabbits: experimental animal study. BMC Complement Altern Med 2011;11:90.

29. Aljadi A, Kamaruddin MY. Evaluation of the phenolic contents and antioxidant capacites of two Malaysan floral honeys. Food Chem 2004;85:513-8.
30. Tonks AJ, Cooper RA, Jones KP, et al. Honey stimulates inflammatory cytokine production from monocytes. Cytokine 2003;21:242-7.

31. Subrahmanyam M. A prospective randomised clinical and histological study of superficial burn wound healing with honey and silver sulfadiazine. Burns 1998;24:157-61.

32. Subrahmanyam M. Topical application of honey in treatment of burns. Br J Surg 1991;78:497-8.

33. Gethin G, et al. Retraction statement: Manuka honey vs. hydrogel - a prospective, open label, multicentre, randomised controlled trial to compare desloughing efficacy and healing outcomes in venous ulcers. J Clin Nurs 2015;24:2686. doi: 10.1111/jocn.12652

34. Molan P, Rhodes T. Honey: a biologic wound dressing. Wound 2015;27:141-51.

35. Weissenstein A, Luchter B, Bittmann S. Medical honey and its role in paediatric patients. Br J Nurs 2014;23:S30-S34.

36. Postrnes T, van der Boogard AE, Hazen M. The sterilization of honey with cobalt 60 gamma radiation: a study of honey spiked with spores of Clostridium botulinum and Bacillus subtilis. Experientia 1995;51:986-9. 